Article

\title{
Characteristics of Mineralization-Forming Fluid and Metallogenic Mechanism for the Mianhuakeng Uranium Deposit in South China: Constraints from In Situ Geochemical Signatures and Sulfur Isotopes of Syn-Mineralization Pyrite and Pitchblende
}

\author{
Qing Lan ${ }^{1}{ }^{*}$, Shanling $\mathrm{Fu}^{1}$ and Jinrong Lin ${ }^{2}$ \\ 1 State Key Laboratory of Ore Deposit Geochemistry, Institute of Geochemistry, Chinese Academy of Sciences, \\ Guiyang 550081, China; fushanling@mail.gyig.ac.cn \\ 2 Beijing Research Institute of Uranium Geology, China National Nuclear Corporation, Beijing 100029, China; \\ linjr68@163.com \\ * Correspondence: lanqing@mail.gyig.ac.cn
}

Citation: Lan, Q.; Fu, S.; Lin, J.

Characteristics of

Mineralization-Forming Fluid and

Metallogenic Mechanism for the

Mianhuakeng Uranium Deposit in

South China: Constraints from In

Situ Geochemical Signatures and

Sulfur Isotopes of Syn-Mineralization

Pyrite and Pitchblende. Minerals 2022,

12, 227. https://doi.org/10.3390/

$\min 12020227$

Academic Editor: David Quirt

Received: 31 December 2021

Accepted: 3 February 2022

Published: 10 February 2022

Publisher's Note: MDPI stays neutral with regard to jurisdictional claims in published maps and institutional affiliations.

Copyright: (C) 2022 by the authors. Licensee MDPI, Basel, Switzerland. This article is an open access article distributed under the terms and conditions of the Creative Commons Attribution (CC BY) license (https:// creativecommons.org/licenses/by/ $4.0 /)$.

\begin{abstract}
The Mianhuakeng deposit is the most representative granite-related hydrothermal veintype uranium deposit in South China; however, the characteristics of the mineralization-forming fluid and metallogenic mechanism are still less constrained. To address the scientific problems above, we investigated the trace element chemistry and sulfur isotope compositions in syn-mineralization pyrite and pitchblende from the Mianhuakeng uranium deposit. The trace element chemistry shows that the mineralization in the Mianhuakeng deposit belongs to an intermediate-to-low temperature hydrothermal system, which is consistent with the homogenization temperature distribution of fluid inclusions. Redox-sensitive elements (such as Co and Se) in syn-mineralization pyrite suggest a reductive nature of the mineralization-forming fluids. The fractionation between light and heavy REE in pitchblende from the Mianhuakeng deposit is most similar to those from the Changjiang pluton. The pronounced negative Eu anomaly is coincident with mineralization-bearing granites. The $\delta^{34} S$ values of syn-mineralization pyrite range from -10.2 to $-1.4 \%$, which is higher than those values of pyrite from granites near the studied area and lower than the $\delta^{34} S$ values of pyrite from diabase in the ore district. The REE signatures of pitchblende and sulfur isotope composition of syn-mineralization pyrite suggest that the major $U$ source for the Mianhuakeng deposit is most likely the Changiiang pluton, probably accompanied by the incorporation of mantle-derived fluids. The circulations of $\mathrm{CO}_{2}$-rich hydrothermal fluids leached uranium from granite source rocks, especially from the Changjiang pluton. The change of physicochemical conditions of the mineralization-forming fluid resulted in the deposition of the uranium minerals in favorable structural traps to form the hydrothermal vein-type Mianhuakeng uranium deposit.
\end{abstract}

Keywords: Mianhuakeng uranium deposit; pyrite and pitchblende; characteristics of mineralizationforming fluid; metallogenic mechanism; South China

\section{Introduction}

South China is well known for its large-scale polymetallic mineralization during the Mesozoic, making the region one of the most important polymetallic metallogenic provinces in the world [1,2]. Of particular importance are world-class deposits of $\mathrm{W}, \mathrm{Sn}$, and Sb [2-5] and some of the largest $\mathrm{Ta}, \mathrm{Cu}, \mathrm{Hg}$, and $\mathrm{U}$ deposits in China $[1,4,6]$. Hydrothermal vein-type uranium mineralization in South China is one of the most important parts of the South China Metallogenic Belt and served as the major source of $U$ resources for the country over the past several decades before the focus of $U$ exploration moved to North China, with the discoveries of sedimentary-hosted $U$ deposits there [2,6,7]. The granite-related 
$\mathrm{U}$ mineralization in South China is one of the most important hydrothermal vein-type deposit types in the region, and it is hosted by Indosinian and Yanshanian granites located in the southeastern part of the Zhuguang complex at the juncture of northern Guangdong, southeastern Hunan, and southwestern Jiangxi provinces (Figures 1 and 2; $[8,9]$ ).

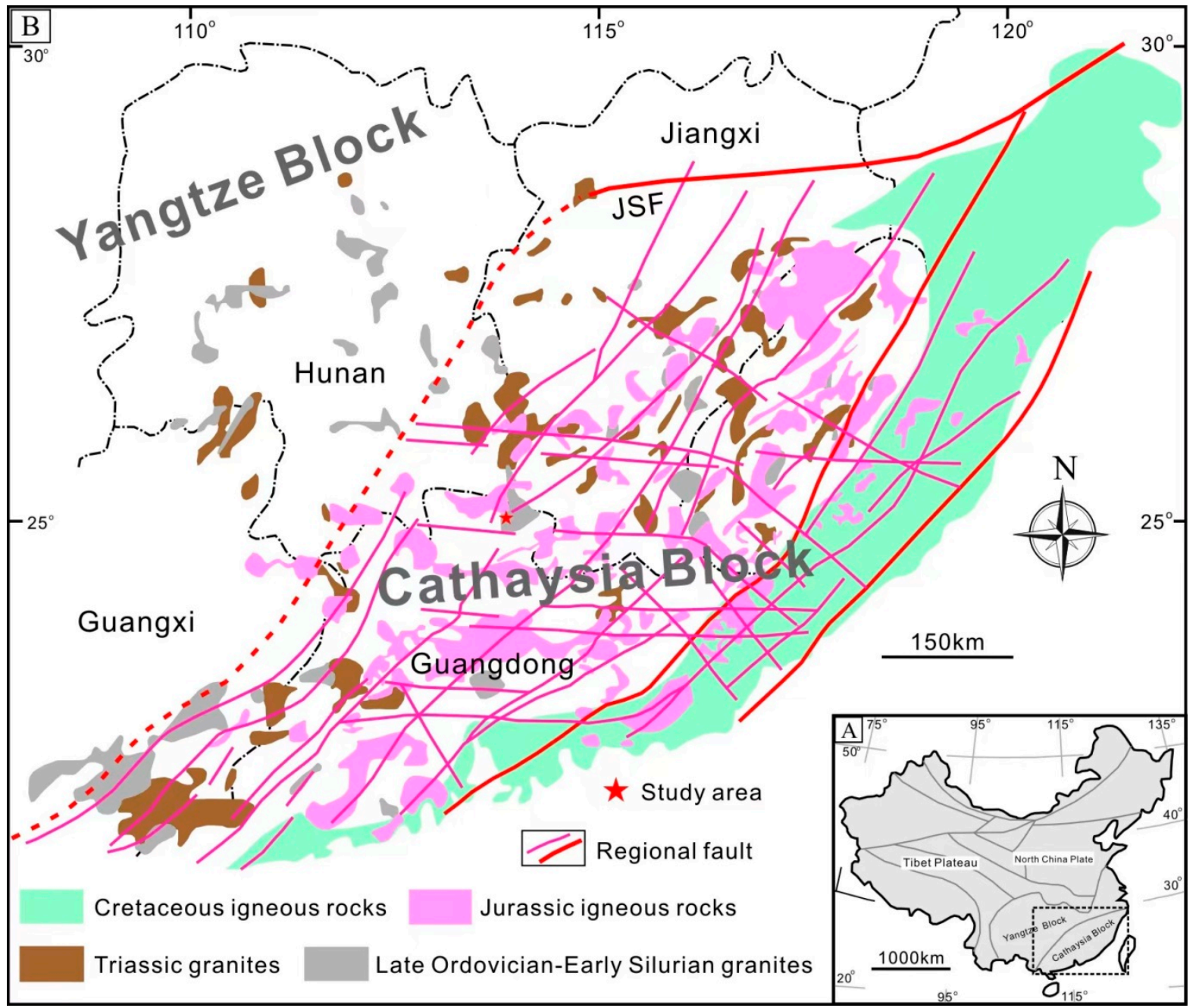

Figure 1. (A) Geological map showing the principal tectonic framework of China. (B) Schematic map of the Cathaysia block showing the structure, igneous rocks, and location of the study area (modified after Sun [10], Li et al. [11] and Lan et al. [12]). Reprinted with permission from ref. [12]. Copyright 2021 Elsevier. JSF: Jiangshan-Shaoxing Fault.

The Mianhuakeng uranium deposit in northern Guangdong province, China, is representative of the granite-related hydrothermal uranium deposits in South China. The mineralization primarily occurred in the contact zone of the Indosinian Youdong pluton and Yanshanian Changjiang pluton (Figure 2). In the past decade, abundant studies have been conducted on this deposit, including the age, geodynamic setting, and the source of mineralizing materials [7,13-20]. However, due to the complexity of the mineralizing system in the Mianhuakeng uranium deposit, the characteristics of the mineralization-forming fluid and metallogenic mechanism are still less well-constrained. In this study, we investigated the trace element and rare earth element (REE) concentrations and sulfur isotope composition in syn-mineralization pyrite and pitchblende from the Mianhuakeng uranium deposit, integrated with the data presented in previous studies. The main purpose of this paper is to discuss the characteristics of the mineralization-forming fluid and metallogenic mechanism for granite-related hydrothermal U deposition in South China. 


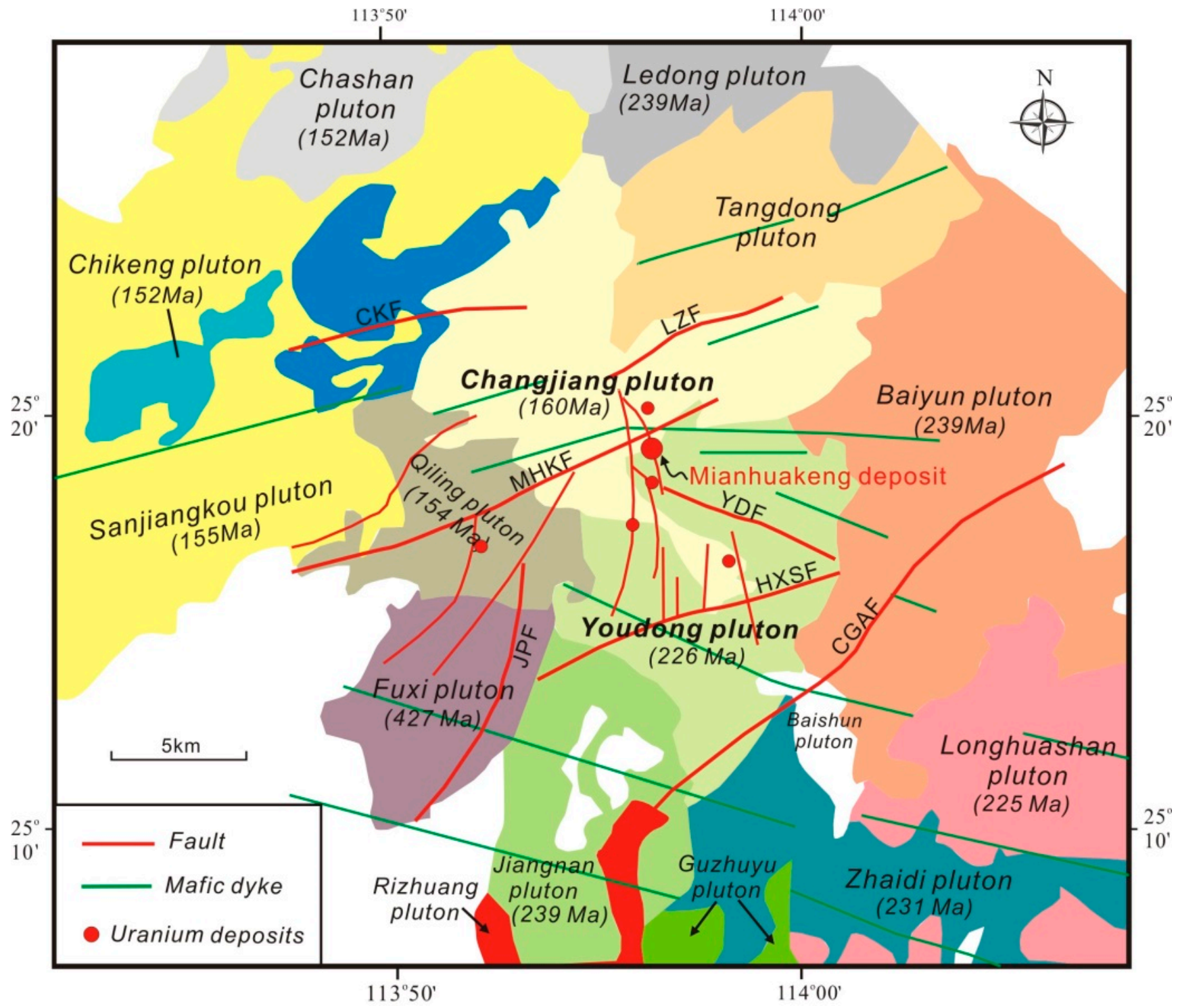

Figure 2. Geological map of the southern Zhuguang granite massif showing the distribution of granites, structure, and the uranium deposits hosted in granites (modified after Zhang et al. [21]; Zhang [22] and Lan et al. [12]). Reprinted with permission from ref. [12]. Copyright 2021 Elsevier. CKF: Chengkou Fault; LZF: Lizhou Fault; MHKF: Mianhuakeng Fault; YDF: Youdong Fault; HXSF: Huangxishui Fault; CGAF: Chenggongao Fault; JPF: Jiaoping Fault.

\section{Regional and Deposit Geology}

The South China Block is made up of the Yangtze block to the northwest and the Cathaysian block to the southeast (Figure 1A), which were amalgamated together along the Jiangshao suture zone at around $830 \mathrm{Ma}[23,24]$. Large amounts of Mesozoic igneous rocks are widely distributed from the coastal region into the continental interior in South China, which is thought to be related to the subduction of the Pacific plate underneath South China $[2,25,26]$. Many U deposits occurred in these Mesozoic granites or volcanic rocks, such as the Zhuguang, Xiangshan, and Xiwang ore fields $[6,9,14]$.

The Changjiang $U$ ore field is very representative of the granite-related $U$ ore fields in South China. The U mineralization primarily occurred in the southeastern part of the Zhuguang complex. Three NE-SW striking regional faults (Lizhou, Mianhuakeng, and Huangxishui faults, from north to south), and one NW-SE striking fault (Youdong fault), 
are the main mineralization-controlling structures. The $\mathrm{N}-\mathrm{S}$ striking faults are the major mineralization-bearing structures (Figure 2).

The Mianhuakeng hydrothermal $U$ deposit is located in the eastern part of the Changjiang uranium ore field. The U mineralization primarily occurred in the contact zone of an Indosinian two-mica granite (Youdong pluton; SHRIMP U-Pb zircon age of $232 \pm 4 \mathrm{Ma}$ ) and an Early Yanshanian biotite granite (Changjiang pluton; U-Pb zircon age of $160 \pm 2 \mathrm{Ma})[8,27,28]$. The mineralization is constrained by the NE-SW-striking Mianhuakeng fault and the NW-SE-striking Youdong fault.

The two-mica granite is widespread in the south and east of the studied area, and generally contains xenomorphic quartz, K-feldspar, plagioclase, biotite, and muscovite, along with accessory minerals including zircon and apatite. The biotite granite is characterized by porphyritic textures with $\mathrm{K}$-feldspar phenocrysts, and its mineral composition is generally quartz, K-feldspar, plagioclase, and biotite, with minor amounts of muscovite. The main mineral species are very similar in these two granites, although their proportions differ $[14,29]$. Hydrothermal alterations present around the U mineralization mainly include hydromicazation (illite), chloritization (chlorite), silicification, fluoritization, sericitization and hematitization. The alteration intensity that the granites underwent was variable with distance from the $\mathrm{U}$ deposits. As a result of the metasomatism, the feldspar and biotite were generally replaced by hydromica (illite) and quartz, and chlorite and hematite, respectively, with hematite occurring in bands along the foliation of the chlorite [14].

The $U$ deposits commonly occur as veins or lenses of quartz-pitchblende in brecciated and altered granite (Figure 3). The vertical range of the $U$ mineralization is large, and minable uranium mineralization is found from the surface down to a depth of ca. $1500 \mathrm{~m}$ [30]. The major $\mathrm{U}$ mineral is fine-grained pitchblende associated with microcrystalline quartz, purple-black fluorite, calcite, and lesser chlorite and sericite. Based on the mineralization textures and paragenetic relationships of the primary minerals, a threestage paragenesis can be identified, namely pre-mineralization, syn-mineralization, and post-miineralization stages (Figure 4). The pre-mineralization stage refers to the hightemperature quartz vein stage that preceded the uranium mineralization. The vein quartz was crushed and then cemented by the microcrystalline quartz in the syn-mineralization stage. Two major types of mineral association were recognized in mineralization from the Mianhuakeng deposit. One is the purple-black fluorite + pyrite + pitchblende + microcrystalline quartz association and the other is the hematite + red microcrystalline quartz + pitchblende association (Figure 5). The pyrite intergrowths with pitchblende generally present in a colloform habit (Figure 5D). The purple-black fluorite is generally distributed in the middle and lower parts of the deposit and has a higher uranium content than the light-colored fluorite in the upper part of the deposit. The post-mineralization stage is characterized by gray-white quartz and calcite, light-colored fluorite, and drusy quartz, suggestive of the end of the hydrothermal activity. 


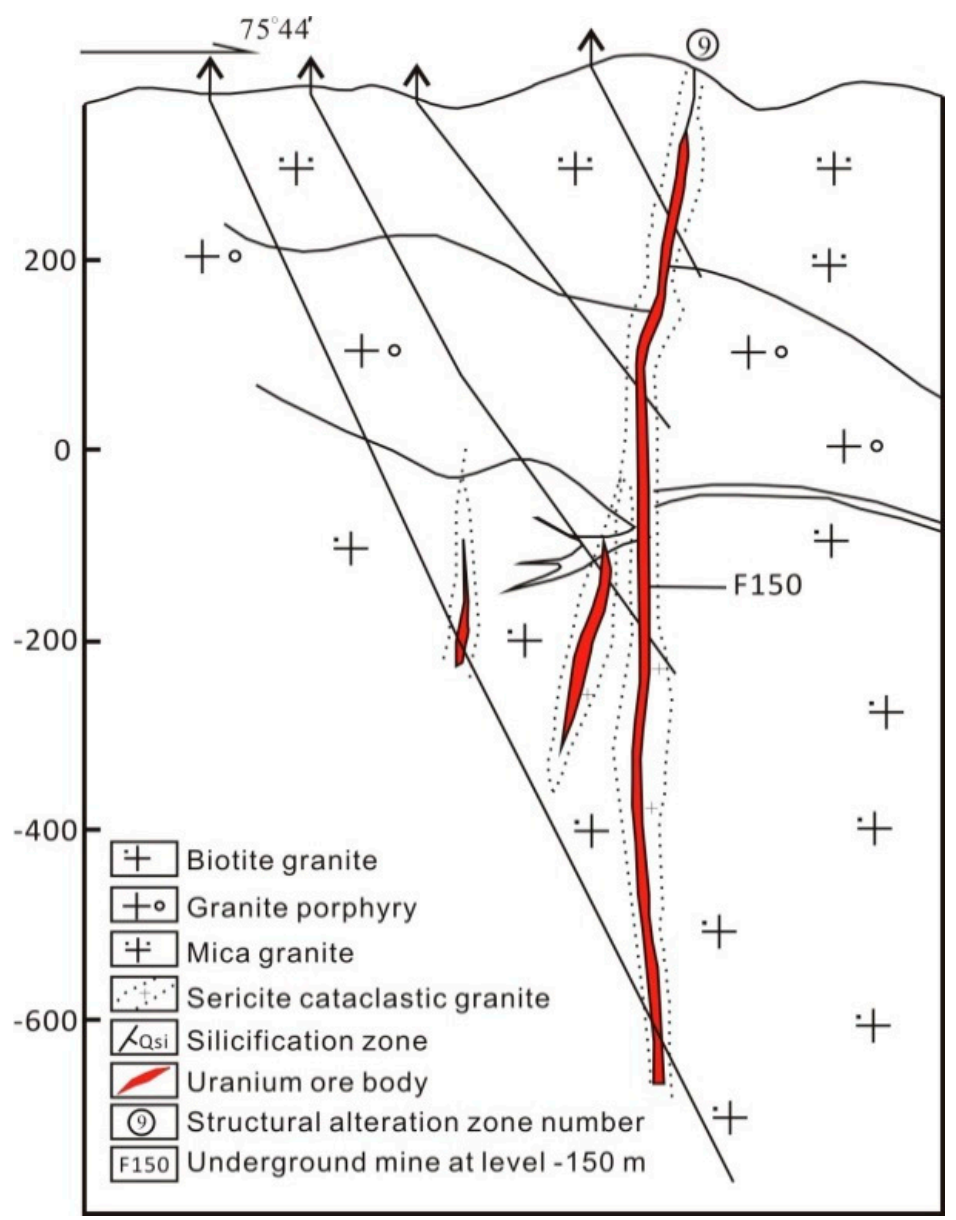

Figure 3. Schematic map of U-mineralized alteration zone No. 9 in the Mianhuakeng U deposit (revised from Qi et al. [19]).

\begin{tabular}{|c|c|c|c|}
\hline Mineral & $\begin{array}{l}\text { Pre-ore } \\
\text { stage }\end{array}$ & Syn-ore stage & Post-ore stage \\
\hline $\begin{array}{l}\text { K-feldspar } \\
\text { Plagioclase } \\
\text { Biotite }\end{array}$ & & & \\
\hline $\begin{array}{l}\text { Pitchblende } \\
\text { Pyrite }\end{array}$ & & & \\
\hline Hematite & & & \\
\hline $\begin{array}{l}\text { Chlorite } \\
\text { Hydromica }\end{array}$ & & & \\
\hline $\begin{array}{l}\text { Fluorite } \\
\text { Calcite }\end{array}$ & & & - \\
\hline
\end{tabular}

Figure 4. Simplified paragenesis of major mineralization and gangue minerals in the Mianhuakeng uranium deposit. 


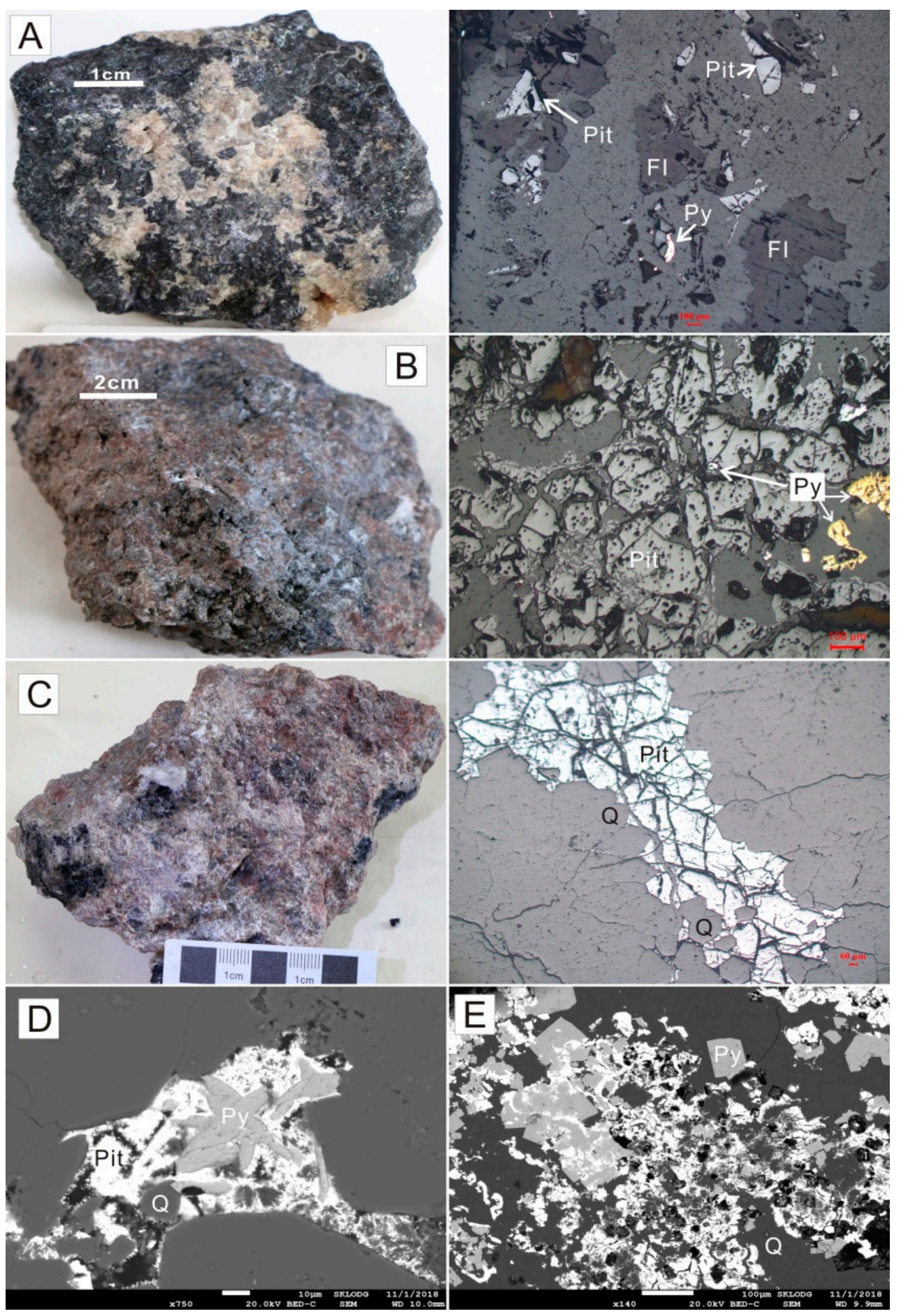

Figure 5. Major types of mineralization in the No. 302 uranium deposit. (A-C) the mineralization from the Mianhuakeng deposit (Left) and its corresponding mineral characteristics under the microscope (Right). (A,B) the mineral association is generally the purple-black fluorite + pyrite + pitchblende + microcrystalline quartz. (C) red ore. The mineral association in this type of mineralization is usually hematite + red microcrystalline quartz + pitchblende. $(\mathbf{D}, \mathbf{E})$ the back-scattered electron images. (D) the syn-mineralization colloform pyrite intergrowth with pitchblende and quartz. (E) the network pitchblende, pyrite and quartz veins. Pit: pitchblende; Fl: fluorite; Py: pyrite; Q: quartz. 


\section{Sampling and Analytical Methods}

The trace element and rare earth element (REE) compositions of pyrite and pitchblende were analyzed in thin sections ( $60 \mu \mathrm{m}$ thick) from three mineralized samples selected from underground mining tunnels at level $-150 \mathrm{~m}$ of the Mianhuakeng deposit. The details of the samples are given in Table 1. While there were only three samples analyzed, they represent the typical metallogenic characteristics of the Mianhuakeng $U$ deposit. A sufficient number of analytical spots on each sample provided suitable analytical data to determine the geochemical signatures of the $\mathrm{U}$ mineralization.

Table 1. The detailed description of mineralization samples collected from the Mianhuakeng uranium deposit.

\begin{tabular}{|c|c|c|c|}
\hline Scheme. & Mineral Assemblage & Textures & Sampling Location \\
\hline MHK08-3 & $\begin{array}{c}\text { purple-black fluorite }+ \text { pyrite }+ \\
\text { pitchblende }+ \text { microcrystalline quartz }\end{array}$ & Massive mineralization & \multirow{3}{*}{$\begin{array}{l}\text { Underground mining tunnels } \\
\text { at level }-150 \mathrm{~m} \text { of the } \\
\text { Mianhuakeng deposit }\end{array}$} \\
\hline MHK07-1 & $\begin{array}{l}\text { hematite }+ \text { red microcrystalline quartz }+ \\
\text { pitchblende }\end{array}$ & Disseminated mineralization & \\
\hline MHK07-2 & $\begin{array}{c}\text { purple-black fluorite }+ \text { pyrite }+ \\
\text { pitchblende }+ \text { microcrystalline quartz }\end{array}$ & Disseminated mineralization & \\
\hline
\end{tabular}

Petrographic observations were conducted on doubly polished thin sections with the optical microscope.

Trace element analyses of pyrite were carried out using laser ablation-inductively coupled-mass spectrometry (LA-ICP-MS) at the State Key Laboratory of Ore Deposit Geochemistry, IGCAS (Guiyang, China). Laser sampling was performed using an ASI RESOLution-LR-S155 laser microprobe equipped with a Coherent Compex-Pro 193 nm ArF excimer laser. An Agilent 7700x ICP-MS instrument was used to acquire ion-signal intensities. Helium $(350 \mathrm{~mL} / \mathrm{min})$ was applied as a carrier gas. The ablated aerosol was mixed with $\operatorname{Ar}(900 \mathrm{~mL} / \mathrm{min})$ as a transport gas, before exiting the cell. Each analysis incorporated a background acquisition of approximately $30 \mathrm{~s}$ (gas blank) followed by $60 \mathrm{~s}$ of data acquisition from the sample. The analysis was run with a $26 \mu \mathrm{m}$ pit size, $5 \mathrm{~Hz}$ pulse frequency and $3 \mathrm{~J} / \mathrm{cm}^{-2}$ fluence. The standard STDGL3 was used to determine concentrations of chalcophile and siderophile elements [31]. The in-house standard pure pyrite Py was used for calibration of the concentrations of $S$ and Fe. The integrated count data to concentrations for lithophile elements were calibrated and converted by GSE-1G and GSD-1G. Sulfide reference material MASS-1 was analyzed as an unknown sample to check the analytical accuracy.

A JEOL JXA-8530F Plus Electron Probe MicroAnalyzer (EPMA) was used to determine the contents of $\mathrm{Si}, \mathrm{Al}, \mathrm{Ti}, \mathrm{K}, \mathrm{Ca}, \mathrm{Fe}$ and $\mathrm{U}$ in quartz using a beam size with a diameter of 1-10 $\mu \mathrm{m}$ and a beam current of $20 \mathrm{nA}$ accelerated at $25 \mathrm{KeV}$ at the SKLODG, Chinese Academy of Sciences (Guiyang, China). Monazite, kaersutite, and $\mathrm{UO}_{2}$ were used as standards for the following elements: $\mathrm{Si}, \mathrm{Al}, \mathrm{Ca}, \mathrm{Fe}, \mathrm{U}$, and Th. The precision of the analyses (at 3б) is $420 \mathrm{ppm}$ for $\mathrm{Si}, 410 \mathrm{ppm}$ for $\mathrm{Al}, 220 \mathrm{ppm}$ for $\mathrm{Ca}, 530 \mathrm{ppm}$ for $\mathrm{Fe}, 540 \mathrm{ppm}$ for $\mathrm{U}$, and $1000 \mathrm{ppm}$ for Th.

In situ analysis of trace element and REE compositions of pitchblende was conducted by LA-ICP-MS at the Analytical Laboratory of the Beijing Research Institute of Uranium Geology. A Coherent Geolas 193 laser ablation system was used in conjunction with a Thermo Scientific High Resolution ICP-MS (HR-ICP-MS). The spot diameter was $16 \mu \mathrm{m}$ with a laser pulse rate of $2 \mathrm{~Hz}$. The energy density was $4 \mathrm{~J} / \mathrm{cm}^{2}$. Helium was used as the carrier gas and merged with argon (make-up gas) via a T-connector before entering the ICP-MS. Each analysis incorporated an approximately $20 \mathrm{~s}$ background acquisition (gas blank) followed by $40 \mathrm{~s}$ data acquisition from the sample. Standards NIST 610, BHVO-2G, BCR-2G, and BIR-1G were used as the external calibration standards. 


\section{Results}

\subsection{Trace Element and Sulfur Isotope Composition of Syn-Mineralization Pyrite}

The data for the LA-ICPMS in situ analysis of trace elements in syn-mineralization pyrite are presented in Table 2. Results show that As concentrations in syn-mineralization pyrite range from 8.86 to 1730.17 ppm, with a majority of analyses being below $300 \mathrm{ppm}$. Te is the third-most abundant trace element in pyrite, after $\mathrm{U}$ and $\mathrm{Pb}$, with contents from 1.92 to $367.46 \mathrm{ppm}$. The $\mathrm{Co}$ and Ni compositions in syn-mineralization pyrite range from 3.15 to $54.32 \mathrm{ppm}$ (average: $26.12 \mathrm{ppm}$ ) and 0.34 to $79.01 \mathrm{ppm}$ (average: $16.31 \mathrm{ppm}$ ), respectively. The Se content varies from 0.28 to $50.46 \mathrm{ppm}$ (average: $10.17 \mathrm{ppm}$ ) and the ratio of Se/Co and Co/Ni ranges from 0.029-11.98 (average 0.704) and 0.09-97.51 (average 9.42), respectively, for the syn-mineralization pyrite. Moreover, Sb has the largest variation of the analyzed trace elements, varying from 0.02 to $347.87 \mathrm{ppm}$ (Figure 6). The U and $\mathrm{Pb}$ compositions are from below detection limit up to $4207.65 \mathrm{ppm}$ and 14,495.5 ppm, respectively. The large variations in concentration suggest that these elements are most likely present in inclusions. The $\delta^{34} \mathrm{~S}$ values of syn-mineralization pyrite range from $-13.2 \%$ to $-1.4 \%$, with an average of $-7.8 \%$ o (Table 3 ).

Table 2. The LA-ICPMS analysis of trace element concentrations (ppm) for syn-mineralization pyrite "_" means "below detection limit".

\begin{tabular}{|c|c|c|c|c|c|c|c|c|c|c|c|}
\hline Samples & As & Se & Co & $\mathrm{Ni}$ & $\mathrm{Sb}$ & $\mathrm{Te}$ & $\mathbf{U}$ & $\mathrm{Pb}$ & Th & Se/Co & $\mathrm{Co} / \mathrm{Ni}$ \\
\hline MHK07-2-01 & 690.08 & 9.82 & 6.81 & 6.37 & - & 21.85 & 1446.48 & 5921.35 & - & 0.32 & 0.09 \\
\hline MHK07-2-02 & 120.56 & 9.48 & - & 4.39 & 0.21 & 42.15 & - & 143.53 & - & - & - \\
\hline MHK07-2-03 & 72.15 & 2.68 & 31.01 & 0.92 & - & 17.87 & 4207.65 & 1050.37 & - & 0.11 & 91.22 \\
\hline МHК07-2-04 & 35.45 & 6.14 & - & 2.75 & 3.78 & 11.48 & 3320.86 & 4170.35 & - & - & - \\
\hline MHK07-2-05 & 98.15 & 1.73 & - & 3.61 & - & 154.11 & - & 6425.65 & - & - & - \\
\hline MHK07-2-06 & 219.45 & 1.74 & 45.75 & 3.47 & 1.03 & 61.48 & - & - & - & 0.07 & 25.30 \\
\hline MHK07-2-07 & 487.01 & 2.38 & 38.73 & 6.58 & - & 125.54 & - & - & - & 0.06 & 1.46 \\
\hline MHK07-2-08 & 120.49 & 0.85 & 4.21 & 5.03 & - & 271.01 & 1242.35 & 5433.67 & - & 11.98 & 0.76 \\
\hline MHK07-2-09 & 810.16 & 15.97 & - & 1.45 & - & 21.11 & - & - & - & - & - \\
\hline MHK07-2-10 & 520.16 & 0.28 & 43.26 & 11.12 & 0.02 & - & 1609.35 & 5121.56 & - & 0.03 & 2.74 \\
\hline MHK07-2-11 & 1730.17 & - & 44.21 & 3.92 & - & 161.58 & 1040.67 & 1735.36 & - & 0.19 & 4.56 \\
\hline MHK07-2-12 & 120.17 & - & 3.15 & 3.03 & - & 35.82 & 3168.55 & 116.54 & - & 1.03 & 0.23 \\
\hline МHК07-2-13 & 1160.06 & 0.78 & 15.48 & 4.88 & - & 345.75 & - & - & - & 0.26 & 12.59 \\
\hline MHK07-2-14 & 50.12 & 3.76 & 5.13 & 43.40 & - & 34.84 & - & 1141.65 & - & 0.24 & 0.24 \\
\hline MHK07-2-15 & 8.86 & 3.46 & 4.81 & 40.50 & - & 69.49 & 247.55 & 733.51 & - & 0.51 & 0.41 \\
\hline MHK07-2-16 & 270.21 & 25.78 & - & 11.50 & - & 162.59 & 1488.96 & 230.25 & - & - & - \\
\hline MHK07-2-17 & 68.46 & 35.15 & - & 8.60 & 20.34 & 27.41 & 3486.61 & 2320.35 & - & - & - \\
\hline MHK07-2-18 & 1470.37 & 32.14 & 19.45 & 26.22 & - & 125.24 & - & - & - & 0.20 & 0.44 \\
\hline MHK07-2-19 & 259.46 & 9.15 & 47.95 & 41.50 & 25.34 & 35.48 & - & 2010.59 & - & 0.83 & 1.64 \\
\hline MHK07-2-20 & 50.26 & 12.25 & 48.28 & 0.39 & 14.86 & 21.47 & - & - & - & 0.18 & 0.67 \\
\hline MHK07-2-21 & 1670.37 & 8.45 & 4.12 & 79.01 & - & 68.73 & - & 335.51 & - & 0.96 & 0.10 \\
\hline MHK07-2-22 & 89.57 & 11.43 & 22.01 & 2.52 & 146.23 & 155.51 & - & - & - & 0.08 & 1.33 \\
\hline MHK07-2-23 & 138.11 & 2.15 & 5.41 & 0.34 & 6.85 & 10.55 & - & 529.53 & - & 0.51 & 0.30 \\
\hline MHK07-2-24 & 490.26 & 11.15 & 23.05 & 3.98 & - & 1.92 & 1249.68 & - & - & 0.43 & 1.63 \\
\hline MHK07-2-25 & 92.34 & 3.48 & - & 0.39 & 12.57 & 19.44 & - & - & - & - & - \\
\hline MHK08-3-1 & 49.58 & 19.32 & 30.77 & 1.81 & - & 38.76 & - & - & - & 0.09 & 4.83 \\
\hline MHK08-3-2 & 154.22 & 6.82 & 43.82 & 26.50 & - & 367.46 & - & - & - & 0.14 & 9.98 \\
\hline МHК08-3-3 & 98.54 & 3.14 & 7.46 & 5.53 & 14.10 & 84.46 & 3252.26 & - & - & 0.23 & 8.10 \\
\hline МHK08-3-4 & 35.49 & 2.46 & 6.28 & 15.40 & 5.17 & 81.57 & 362.65 & $11,690.00$ & - & 0.28 & 2.28 \\
\hline MHK08-3-5 & 410.12 & 50.46 & 39.45 & 15.81 & - & 35.46 & - & - & - & 0.06 & 10.93 \\
\hline МHК08-3-6 & 241.55 & 22.49 & 24.83 & 9.70 & 347.84 & 225.55 & - & 50.35 & - & 0.03 & 7.16 \\
\hline MHK08-3-7 & 390.15 & 1.24 & 48.31 & 14.00 & - & 27.16 & - & - & - & 0.33 & 7.34 \\
\hline МHK08-3-8 & 20.15 & 8.19 & 7.16 & 1.23 & - & 135.58 & 1392.36 & - & - & 0.04 & 1.43 \\
\hline MHK08-3-9 & 320.04 & 3.25 & - & 21.70 & 17.96 & 34.54 & 435.58 & - & - & - & - \\
\hline МHK08-3-10 & 67.48 & 3.95 & 35.65 & 11.66 & 111.46 & - & - & $14,492.35$ & - & - & 3.21 \\
\hline
\end{tabular}


Table 2. Cont.

\begin{tabular}{|c|c|c|c|c|c|c|c|c|c|c|c|}
\hline Samples & As & Se & Co & $\mathbf{N i}$ & $\mathrm{Sb}$ & $\mathrm{Te}$ & $\mathbf{U}$ & $\mathrm{Pb}$ & Th & Se/Co & $\mathrm{Co} / \mathrm{Ni}$ \\
\hline MHK08-3-11 & 150.54 & 1.25 & 6.56 & 6.48 & 0.28 & 285.02 & - & 1783.55 & - & 0.12 & 1.67 \\
\hline МHК08-3-12 & 159.58 & 2.43 & 22.12 & 37.30 & - & 321.56 & - & 1540.32 & - & 0.17 & 7.30 \\
\hline MHK08-3-13 & 79.85 & 27.82 & 54.32 & 44.00 & 14.75 & 65.05 & 2033.42 & 1610.07 & - & 0.06 & 11.13 \\
\hline МHК08-3-14 & 35.48 & 13.15 & 9.05 & 29.26 & - & 41.75 & - & - & - & 2.85 & 0.21 \\
\hline MHK08-3-15 & 348.53 & 3.83 & - & 72.30 & - & 165.25 & 2445.95 & 3050.27 & - & - & - \\
\hline MHK08-3-16 & 690.21 & 39.96 & 46.03 & 40.70 & - & 27.26 & - & 2152.36 & - & 0.70 & 4.00 \\
\hline МНК08-3-17 & 44.02 & 8.88 & 48.58 & 16.52 & 23.21 & 61.60 & - & - & - & 0.19 & 5.65 \\
\hline MHК08-3-18 & 240.54 & 3.96 & 33.06 & 17.77 & 292.26 & 99.17 & - & 1768.75 & - & 0.37 & 1.26 \\
\hline MHК08-3-19 & 127.95 & 1.74 & - & 14.15 & - & 157.15 & - & 20.65 & - & - & - \\
\hline MHK08-3-20 & 157.61 & 2.74 & 38.03 & 16.13 & - & 198.16 & 1646.86 & 2365.93 & - & 0.30 & 97.51 \\
\hline
\end{tabular}

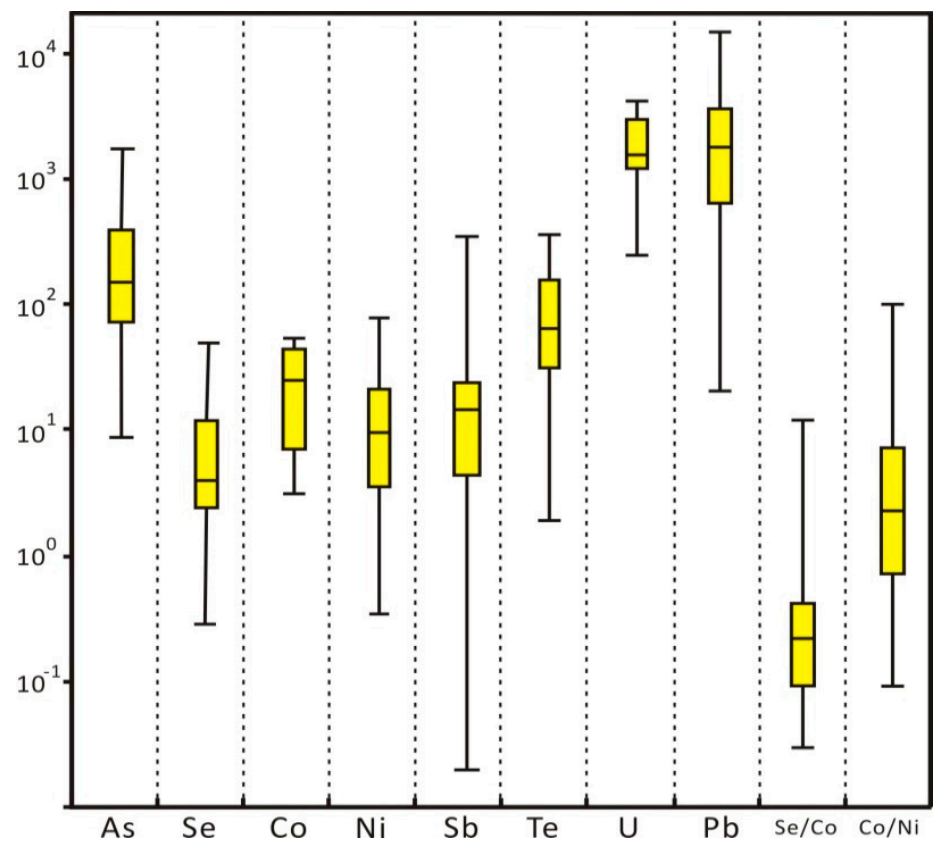

Figure 6. Box diagram of trace element concentrations and ratios for the syn-mineralization pyrite from the Mianhuakeng U deposit. Concentrations are in ppm.

Table 3. The $\delta^{34} \mathrm{~S}$ values for syn-mineralization pyrite from the Mianhuakeng $U$ deposit.

\begin{tabular}{cccc}
\hline No. & Sample & Mineral & $\boldsymbol{\delta}^{\mathbf{3 4}} \mathbf{S} \mathbf{( \% )}$ \\
\hline 1 & ZK83-1 & Pyrite & -10.1 \\
2 & ZK83-2 & Pyrite & -3.7 \\
3 & ZK83-3 & Pyrite & -13.2 \\
4 & ZK83-4 & Pyrite & -8.9 \\
5 & ZK83-5 & Pyrite & -9.8 \\
6 & ZK83-6 & Pyrite & -10.2 \\
7 & ZK83-7 & Pyrite & -1.4 \\
8 & ZK83-8 & Pyrite & -9.5 \\
9 & MHK07-2-1 & Pyrite & -6.5 \\
10 & MHK07-2-2 & Pyrite & -7.8 \\
11 & MHK07-2-3 & Pyrite & -9.5 \\
12 & MHK07-2-4 & Pyrite & -7.8 \\
13 & MHK07-2-5 & Pyrite & -6.4 \\
14 & MHK07-2-6 & Pyrite & -4.6 \\
\hline
\end{tabular}




\subsection{Trace Elements and REE Characteristics of Pitchblende}

The EMPA and LA-ICP-MS analytical results for trace elements and REE of pitchblende are presented in Tables 4 and 5, respectively. The compositions of pitchblende from the Mianhuakeng $\mathrm{U}$ deposit generally show variable amounts of $\mathrm{SiO}_{2}$, varying from 1.49 to $5.13 \mathrm{wt} \%$, and relatively high $\mathrm{CaO}(8.4 \sim 11.0 \mathrm{wt} \%)$. The $\mathrm{CaO}$ content in each sample is relatively homogeneous, thus it was most likely incorporated during crystallization, although a minor part related to post-crystallization alteration cannot be excluded. Most of the analysis spots have $\mathrm{SiO}_{2}$ concentrations $<3 \mathrm{wt} \%$ suggesting limited post-crystallization alteration. The $\mathrm{UO}_{2}$ concentrations in pitchblende are from 68.99 to $82.57 \mathrm{wt} \%$, while there are only low concentrations of fluorine, ranging from below detection limits (BDL) to 0.71 $\mathrm{wt} \% \mathrm{~F}$. In addition, high concentrations of W (up to $2395 \mathrm{ppm}$ ) and Y (up to $3884 \mathrm{ppm}$ ), and extremely low amounts of Th (from BDL to $0.27 \mathrm{ppm}$ ) and $\mathrm{Zr}$ (most of the spots are $\mathrm{BDL}$ ) are present in the pitchblende. A large variation of $\mathrm{Nb}$ concentration (from BDL to $515 \mathrm{ppm}$ ) is also present in the $\mathrm{U}$ oxide samples. Pitchblende has high and relatively homogeneous lead contents that range from 1.02 to $3.83 \mathrm{wt} \%$.

Table 4. Representative EMPA analyses (wt\%) of pitchblende. "/" means "below detection limit".

\begin{tabular}{|c|c|c|c|c|c|c|c|c|c|}
\hline No. & Samples & $\mathrm{UO}_{2}$ & $\mathrm{Al}_{2} \mathrm{O}_{3}$ & $\mathrm{SiO}_{2}$ & $\mathrm{CaO}$ & $\mathrm{MnO}$ & $\mathrm{FeO}$ & $\mathbf{F}$ & Th \\
\hline 1 & MHK08-3-1 & 81.06 & 0.24 & 2.71 & 8.70 & 0.47 & 0.35 & 0.54 & / \\
\hline 2 & MHK08-3-2 & 77.74 & 0.44 & 3.33 & 9.05 & 0.80 & 0.67 & 0.54 & / \\
\hline 3 & MHK08-3-3 & 78.82 & 0.36 & 3.06 & 9.48 & 0.63 & 0.41 & 0.71 & / \\
\hline 4 & MHK08-3-4 & 78.02 & 0.11 & 2.56 & 10.20 & 0.77 & 0.38 & 0.42 & / \\
\hline 5 & MHK08-3-5 & 68.99 & 0.32 & 2.73 & 9.15 & 0.71 & 0.34 & 0.19 & / \\
\hline 6 & MHK08-3-6 & 77.32 & 0.39 & 1.89 & 10.20 & 0.72 & 0.34 & 0.30 & / \\
\hline 7 & MHK08-3-7 & 77.09 & 0.42 & 2.52 & 10.90 & 0.79 & 0.47 & / & / \\
\hline 8 & MHK08-3-8 & 77.91 & 0.4 & 2.89 & 10.40 & 0.6 & 0.32 & 0.17 & / \\
\hline 9 & MHK08-3-9 & 74.05 & 0.46 & 5.13 & 8.80 & 0.70 & 0.50 & 0.18 & / \\
\hline 10 & MHK08-3-10 & 78.38 & 0.44 & 2.86 & 9.56 & 0.73 & 0.41 & 0.37 & / \\
\hline 11 & MHK07-1-1 & 82.57 & / & 1.49 & 8.80 & 0.83 & 0.52 & 0.42 & / \\
\hline 12 & MHK07-1-2 & 76.23 & 0.6 & 4.24 & 8.43 & 0.87 & 0.44 & 0.38 & / \\
\hline 13 & MHK07-1-3 & 77.44 & 0.32 & 2.58 & 10.80 & 0.69 & 0.34 & / & / \\
\hline 14 & MHK07-1-4 & 78.01 & 0.46 & 3.23 & 9.55 & 0.77 & 0.47 & 0.17 & / \\
\hline 15 & MHK07-1-5 & 79.24 & 0.37 & 3.01 & 9.65 & 0.62 & 0.39 & 0.49 & / \\
\hline 16 & MHK07-1-6 & 78.22 & 0.41 & 2.41 & 10.20 & 0.63 & 0.37 & 0.47 & / \\
\hline 17 & MHK07-1-7 & 76.47 & 0.40 & 2.70 & 11.00 & 0.50 & 0.36 & 0.21 & / \\
\hline 18 & MHK07-1-8 & 78.34 & 0.45 & 2.92 & 10.60 & 0.49 & 0.27 & 0.37 & / \\
\hline 19 & MHK07-1-9 & 77.11 & 0.48 & 4.30 & 9.97 & 0.48 & 0.24 & 0.38 & / \\
\hline
\end{tabular}

The total REE contents in pitchblende ( $\mathrm{REE}$ ) vary from 868 to 7798 ppm, with an average of $3171 \mathrm{ppm}$. The fractionation of light and heavy REE is shown by the ratio of $\sum$ LREE/ $\sum$ HREE that ranges from 1.7 to 10.0 (average 5.4) in all sample spots. The values of $(\mathrm{La} / \mathrm{Yb})_{\mathrm{N}}$ vary from 0.80 to 15.62 (average 7.07) for sample MHK08-3 and from 2.68 to 43.51 (average 13.78) for sample MHK07-1. The $(\mathrm{La} / \mathrm{Sm})_{\mathrm{N}}$ values range from 0.72 to 12.81 for both samples. The $(\mathrm{Gd} / \mathrm{Lu})_{\mathrm{N}}$ values of all sample spots vary from 1.05 to 3.68. The $\mathrm{Eu} / \mathrm{Eu}^{*}$ values range from 0.30 to 0.61 (average 0.40 ) for Mianhuakeng pitchblende, indicating a pronounced negative Eu anomaly. 
Table 5. The LA-ICPMS analyses of trace element and REE concentrations (ppm) for pitchblende. "/" means "below detection limit".

\begin{tabular}{|c|c|c|c|c|c|c|c|c|c|c|c|c|c|c|c|c|c|c|}
\hline No. & Sample No. & $\mathrm{V}$ & $\mathrm{Cu}$ & $\mathrm{Ga}$ & $\mathbf{R b}$ & $\mathrm{Sr}$ & $Y$ & $\mathrm{Zr}$ & $\mathrm{Nb}$ & Mo & Ba & W & $\mathrm{Tl}$ & $\mathrm{Pb}$ & Th & La & Ce & Pr \\
\hline 1 & MHK08-3-1 & 122 & 10.00 & 12.2 & 4.57 & 184 & 960 & / & 0.30 & / & 59.8 & 1830 & 1.27 & 11,388 & 0.11 & 959 & 773 & 90.6 \\
\hline 2 & MHK08-3-2 & 81.2 & 14.00 & 40.0 & 4.66 & 162 & 3787 & / & 515 & 0.82 & 38.6 & 2208 & 2.29 & 11,594 & 0.04 & 525 & 1660 & 252.0 \\
\hline 3 & MHK08-3-3 & 101.0 & / & 23.3 & 18.2 & 188 & 1550 & I & 71.9 & 24.30 & 48.2 & 2382 & 1.46 & 13,170 & 0.02 & 345 & 703 & 97.3 \\
\hline 4 & MHK08-3-4 & 101.0 & 8.84 & 21.3 & / & 133 & 2619 & / & 91.9 & 4.54 & 45.2 & 1900 & 0.86 & 12,980 & 0.10 & 456 & 1224 & 179.0 \\
\hline 5 & MHK08-3-5 & 104.0 & / & 13.3 & / & 136 & 837 & / & 8.75 & 3.65 & 41.2 & 1777 & 8.12 & 38,282 & / & 344 & 566 & 76.4 \\
\hline 6 & MHK08-3-6 & 115.0 & 15.50 & 11.4 & / & 144 & 992 & 7.69 & 2.66 & 3.14 & 43.1 & 1878 & 1.96 & 17,084 & 0.06 & 373 & 602 & 79.4 \\
\hline 7 & MHK08-3-7 & 114.0 & 8.51 & 8.17 & / & 154 & 428 & / & 1.61 & 7.70 & 49.4 & 1986 & 1.44 & 14,728 & 0.01 & 253 & 247 & 36.8 \\
\hline 8 & MHK08-3-8 & 111.0 & / & 19.0 & / & 149 & 878 & / & 3.72 & 1.87 & 48.9 & 1793 & 3.13 & 14,208 & 0.08 & 554 & 796 & 89.4 \\
\hline 9 & MHK08-3-9 & 105.0 & 84.80 & 15.0 & / & 155 & 903 & 25.60 & 7.43 & 2.55 & 35.1 & 1790 & 4.95 & 18,392 & 0.27 & 488 & 813 & 102.0 \\
\hline 10 & MHK08-3-10 & 110.0 & 7.78 & 17.3 & / & 143 & 778 & / & 1.96 & 2.16 & 30.5 & 1867 & 1.43 & 14,645 & 0.04 & 538 & 821 & 88.5 \\
\hline 12 & MHK07-1-2 & 91.8 & 11.10 & 9.29 & / & 180 & 513 & / & / & 4.24 & 44.4 & 1960 & 4.55 & 18,787 & 0.01 & 413 & 473 & 48.1 \\
\hline 13 & MHK07-1-3 & 78.6 & 21.10 & 58.0 & 28.6 & 147 & 3884 & / & 39.4 & 8.42 & 41.2 & 2161 & 0.93 & 11,289 & 0.15 & 1054 & 2654 & 367.0 \\
\hline 14 & MHK07-1-4 & 95.6 & 2.67 & 9.53 & 23.9 & 157 & 749 & / & 1.16 & / & 46.1 & 1885 & 0.90 & 13,949 & / & 537 & 574 & 55.6 \\
\hline 15 & MHK07-1-5 & 84.8 & / & 26.9 & 6.59 & 162 & 2095 & / & 7.47 & / & 31.6 & 1940 & 1.54 & 11,460 & 0.09 & 784 & 1377 & 151.0 \\
\hline 16 & MHK07-1-6 & 80.5 & 26.10 & 38.1 & / & 153 & 3217 & / & 18.6 & / & 33.9 & 1898 & 2.91 & 11,501 & 0.11 & 868 & 1890 & 235.0 \\
\hline 17 & MHK07-1-7 & 89.0 & 14.90 & 5.97 & 12.6 & 145 & 565 & 4.89 & 2.14 & / & 46.5 & 1787 & 2.44 & 17,565 & / & 517 & 436 & 36.4 \\
\hline 18 & MHK07-1-8 & 89.3 & 15.80 & 22.2 & 18.2 & 159 & 2359 & / & 7.21 & 6.69 & 43.8 & 2031 & / & 10,721 & 0.07 & 958 & 1729 & 214.0 \\
\hline 19 & MHK07-1-9 & 91.1 & 26.40 & 45.2 & 19.2 & 169 & 3072 & I & 5.31 & 5.08 & 59.9 & 2042 & 2.79 & 11,610 & 0.12 & 885 & 1886 & 245.0 \\
\hline No. & Sample No. & $\mathrm{Nd}$ & $\mathrm{Sm}$ & Eu & Gd & $\mathbf{T b}$ & Dy & Ho & Er & $\mathrm{Tm}$ & $\mathbf{Y b}$ & Lu & $\sum \mathrm{REE}$ & $\begin{array}{l}\text { LREE/ } \\
\text { HREE }\end{array}$ & $(\mathrm{La} / \mathrm{Yb})_{\mathrm{N}}$ & $\mathrm{Eu} / \mathbf{E u}^{*}$ & $(\mathrm{La} / \mathrm{Sm})_{\mathrm{N}}$ & $(\mathrm{Gd} / \mathrm{Lu})_{\mathrm{N}}$ \\
\hline 2 & MHK08-3-2 & 1123 & 459.0 & 84.2 & 495 & 108 & 706 & 142 & 400 & 60.7 & 443 & 58.7 & 6516.60 & 1.70 & 0.80 & 0.54 & 0.72 & 1.05 \\
\hline 3 & MHK08-3-3 & 433 & 153.0 & 34.6 & 195 & 37.8 & 255 & 51.7 & 149 & 19.8 & 148 & 20.9 & 2643.10 & 2.01 & 1.58 & 0.61 & 1.42 & 1.16 \\
\hline 4 & MHK08-3-4 & 784 & 286.0 & 41.2 & 320 & 56.3 & 363 & 75.4 & 198 & 30.7 & 184 & 26.9 & 4224.50 & 2.37 & 1.67 & 0.41 & 1.00 & 1.48 \\
\hline 5 & MHK08-3-5 & 316 & 92.6 & 12.3 & 105 & 15.8 & 101 & 19.9 & 51.5 & 7.44 & 45 & 7.27 & 1760.21 & 3.99 & 5.17 & 0.38 & 2.34 & 1.80 \\
\hline 6 & MHK08-3-6 & 339 & 99.1 & 13.2 & 123 & 18.0 & 127 & 25.4 & 70.8 & 9.65 & 65.2 & 8.44 & 1953.19 & 3.36 & 3.87 & 0.37 & 2.37 & 1.81 \\
\hline 7 & MHK08-3-7 & 149 & 38.3 & 5.7 & 43 & 6.41 & 36.7 & 7.81 & 21.1 & 2.48 & 18.5 & 2.57 & 868.37 & 5.27 & 9.24 & 0.43 & 4.16 & 2.08 \\
\hline 8 & MHK08-3-8 & 311 & 68.8 & 7.73 & 89.4 & 12.0 & 66.5 & 13.8 & 35.7 & 5.5 & 30.6 & 4.68 & 2085.11 & 7.08 & 12.23 & 0.30 & 5.07 & 2.38 \\
\hline 9 & MHK08-3-9 & 375 & 86.5 & 12.3 & 104 & 14.8 & 87.7 & 15.7 & 46.7 & 6.19 & 44.4 & 5.94 & 2202.23 & 5.77 & 7.43 & 0.40 & 3.55 & 2.18 \\
\hline 10 & MHK08-3-10 & 353 & 62.3 & 8.14 & 86.6 & 10.5 & 64 & 12.3 & 32.2 & 4.44 & 27.7 & 4.42 & 2113.10 & 7.73 & 13.12 & 0.34 & 5.44 & 2.44 \\
\hline 11 & MHK07-1-1 & 299 & 60.4 & 7.54 & 69.9 & 9.29 & 57.1 & 11.6 & 31.2 & 3.98 & 27.0 & 3.62 & 1879.83 & 7.80 & 12.64 & 0.35 & 5.26 & 2.40 \\
\hline 12 & MHK07-1-2 & 173 & 33.1 & 4.02 & 50.5 & 4.43 & 28.8 & 5.23 & 13.7 & 1.61 & 11.1 & 2.2 & 1261.79 & 9.73 & 25.14 & 0.30 & 7.85 & 2.86 \\
\hline 13 & MHK07-1-3 & 1533 & 426.0 & 47.1 & 479 & 73.9 & 467 & 95.4 & 261 & 38 & 266 & 36.5 & 7797.90 & 3.54 & 2.68 & 0.32 & 1.56 & 1.63 \\
\hline 14 & MHK07-1-4 & 200 & 44.5 & 7.72 & 65.8 & 8.13 & 44.4 & 9.35 & 31.4 & 3.31 & 19.2 & 2.44 & 1602.85 & 7.71 & 18.90 & 0.44 & 7.60 & 3.36 \\
\hline 15 & MHK07-1-5 & 590 & 140.0 & 19.5 & 201 & 29.0 & 165 & 35.1 & 91.4 & 11.3 & 70.1 & 12.2 & 3676.60 & 4.98 & 7.56 & 0.35 & 3.52 & 2.05 \\
\hline 16 & MHK07-1-6 & 1024 & 265.0 & 42.2 & 371 & 55.8 & 341 & 70.1 & 200 & 29.2 & 181 & 25.5 & 5597.80 & 3.40 & 3.24 & 0.41 & 2.06 & 1.81 \\
\hline 17 & MHK07-1-7 & 123 & 25.4 & 5.29 & 48.5 & 5.22 & 28.4 & 5.81 & 14.8 & 1.89 & 8.03 & 1.64 & 1257.38 & 10.00 & 43.51 & 0.45 & 12.81 & 3.68 \\
\hline 18 & MHK07-1-8 & 829 & 213.0 & 29.1 & 264 & 38.2 & 229 & 46.5 & 125 & 18.5 & 110 & 17.4 & 4820.70 & 4.68 & 5.89 & 0.37 & 2.83 & 1.89 \\
\hline 19 & MHK07-1-9 & 1010 & 268.0 & 37 & 350 & 52.8 & 311 & 58.8 & 154 & 21.8 & 134 & 20.0 & 5433.40 & 3.93 & 4.46 & 0.37 & 2.08 & 2.18 \\
\hline
\end{tabular}


Being closely spatially related to mineralization, the mineralization-bearing Indosinian Youdong pluton, the Yanshanian Changjiang pluton, and the mafic dikes in the ore district are used as a comparison $[27,29,32]$. The mineralization-bearing Indosinian Youdong pluton and Yanshanian Changjiang pluton have the LREE/HREE and $(\mathrm{La} / \mathrm{Yb})_{\mathrm{N}}$ values ranging from 8.56 to 11.53 (average 9.50) and 10.47 to 18.82 (average 13.59), 5.81 to 9.75 (average 7.74 ) and 5.23 to 11.91 (average 8.36), respectively. The mafic dikes have LREE/HREE and $(\mathrm{La} / \mathrm{Yb})_{\mathrm{N}}$ values varying from 6.29 to 6.97 (average 6.56) and 6.97 to 8.89 (average 7.76), respectively. The $(\mathrm{La} / \mathrm{Sm})_{\mathrm{N}}$ and $(\mathrm{Gd} / \mathrm{Lu})_{\mathrm{N}}$ values in the Youdong and Changjiang plutons, and in the mafic dikes are from 2.73 to 3.24 and 2.54 to $4.09,2.76$ to 3.85 and 1.21 to 2.59, 2.39 to 3.04 and 2.00 to 2.57, respectively. Pronounced negative Eu anomalies are present in both of the mineralization-bearing granites, with $\mathrm{Eu} / \mathrm{Eu}^{*}$ values ranging from 0.15 to 0.26 for the Youdong pluton and from 0.17 to 0.35 for the Changjiang pluton. However, the mafic dikes have $\mathrm{Eu} / \mathrm{Eu}^{*}$ values ranging from 0.83 to 1.00 .

\section{Discussion}

\subsection{The Characteristics of the Mineralization-Forming Fluid}

Pyrite is the most abundant sulfide in the Earth's crust and is also a major constituent of hydrothermal mineralization in a wide variety of ore systems. Moreover, pyrite can contain minor elements such as $\mathrm{As}, \mathrm{Au}, \mathrm{Cu}, \mathrm{Pb}, \mathrm{Zn}, \mathrm{Co}, \mathrm{Ni}, \mathrm{Sb}, \mathrm{Se}, \mathrm{Te}$, and Hg [33-38]. Consequently, pyrite is not only a sink for iron and sulfur, but it also plays an important role in recording the characteristics of the mineralization-forming fluid and its evolution. Thus, the investigation of the abundance of trace elements in pyrite can provide robust constraints on how ore deposits are formed and is widely used to characterize the properties of mineralization-forming fluids [34,36,38,39].

As sulfur and arsenic display similar geochemical properties, the substitution of As for $S$ is common in pyrite. Previous studies have shown that low temperatures of formation facilitate the substitution of As in pyrite [38,40]. High As contents (up to thousands ppm) are usually only observed in pyrite formed under low-temperature environment $\left(<250{ }^{\circ} \mathrm{C}\right)$, but As contents are usually below 100 ppm in a high-temperature system (e.g., porphyry copper system) [38,41,42]. The As contents in Mianhuakeng syn-mineralization pyrite show a large variation from 8.86 to $1730.17 \mathrm{ppm}$, with an average of $325.87 \mathrm{ppm}$ (Figure 6). This range lies in the range typical of intermediate to low temperature pyrite. It is also consistent with the homogenization temperature distribution of fluid inclusions (between 242 and $140{ }^{\circ} \mathrm{C}$ ) [16-19]. Goodfellow et al. [43] concluded that $\mathrm{Co}, \mathrm{Ni}, \mathrm{Cu}$, and Mo in pyrite are generally of hydrothermal origin. Different primary Co and Ni concentrations in fluids and their preferential incorporation into pyrite are a function of fluid temperatures and $f_{\mathrm{S} 2}$. In a hydrothermal system, the variable temperature likely has a greater influence on the content of $\mathrm{Co}$ and $\mathrm{Ni}$ in pyrite. The ratio of $\mathrm{Co}$ and $\mathrm{Ni}$ in syn-mineralization pyrite from the Mianhuakeng deposit varies from 0.09 to 97.51 (average 9.42) (Table 2 and Figure 6), which are similar to the those of intermediate to low temperature pyrite [44]. As Te is usually enriched in low-temperature pyrite (up to 100 10,000 ppm) [38], the Te content can be diagnostic of formation temperature. In syn-mineralization pyrite Te ranges from 1.92 to $367.46 \mathrm{ppm}$ (average $103.6 \mathrm{ppm}$ ), suggesting that the Mianhuakeng $U$ mineralization belongs to an intermediate to low temperature hydrothermal system.

The geochemical nature of the various REEs is similar and they generally transport as a whole in geological processes. As the ionic radii of the REE (1.16-0.977 $\AA$ ) are close to that of $U^{4+}(1 \AA)$, it is relatively easy for the REEs to substitute for $U$ during mineral crystallization, and the REE contents are little modified by post-crystallization events [45]. The total REE content in U oxides decreases and a fractionation occurs between REEs ( $\sum$ LREE $/ \sum$ HREE $>1$ ) due to the crystallographic control of the mineral structure [45]. The $\sum$ REE in pitchblende ranges from 868 to $7798 \mathrm{ppm}$ and the $\sum \mathrm{LREE} / \sum \mathrm{HREE}$ values range from 1.7 to 10.0 (average 5.39) in all samples (Table 5). Moreover, most of the analyzed spots in the pitchblende have very low Th concentrations ranging from BDL to $0.27 \mathrm{ppm}$, indicative of low-temperature formation. Therefore, the low total REE concentrations 
(below $1 \mathrm{wt} . \%)$ and extremely low Th $(\leq 0.3 \mathrm{ppm})$ contents in the pitchblende grains are also suggestive of a typical low to intermediate temperature hydrothermal deposit, which is consistent with the temperatures obtained from fluid inclusions.

The redox nature of the mineralization system is very important for understanding the evolution of mineralization-forming fluid and the precipitation mechanism of the deposit elements. The variable valence metal elements would be transported by different valence and different complexes in fluids, with reducing nature versus an oxidizing nature; consequently, they would have different geochemical properties. Both Se and Co are redox-sensitive, but in opposite directions, such that the ratio Se/Co in pyrite becomes an extremely sensitive redox proxy [37]. The Se/Co ratios in the Mianhuakeng pyrite range from 0.03 to 11.98 , with an average of 0.70 , suggesting the reducing nature of the mineralization-forming fluid.

Uranium is also a variable valence element, and the $\mathrm{U}^{4+}$ and $\mathrm{U}^{6+}$ compounds are stable in various geological process [30]. Some studies suggest that $U$ is transported in aqueous fluid in the form of $\mathrm{U}^{6+}$ complexes and that it was reduced to $\mathrm{U}^{4+}$ and precipitated as pitchblende during mineralization $[18,46]$. However, in recent years, the research advances in hydrothermal uranium mineralization show that the mineralization-forming fluid is reducing and the $\mathrm{U}$ transport was in the form of $\mathrm{U}^{4+}$ complexes, and also that $\mathrm{U}$ precipitated as a result of the changes of physicochemical conditions (such as the large fluctuations in $\mathrm{pH}$ ) in the mineralization-forming fluid [12,30], which is consistent with the Se and Co observations noted above. A negative Eu anomaly is generally visible for vein-type deposits and was caused by the reducing conditions during the precipitation of the uranium oxides, although other processes may also influence REE incorporation $[45,47]$. A pronounced negative Eu anomaly in both samples from the Mianhuakeng deposit may also support the interpretation that the mineralization-forming fluid was reducing in character.

\subsection{The Metallogenic Mechanism for the Mianhuakeng U Deposit}

The fractionation of REEs in U oxides is less sensitive to redox changes, and it is confirmed that no major REE fractionation occurred from source to $\mathrm{U}$ oxide crystallization at Mianhuakeng. Therefore, the REE signatures of $U$ oxides can be used to identify the most favorable $U$ sources for the granite-related hydrothermal mineralization [45].

The REE patterns provided by pitchblende from the Mianhuakeng deposit are consistent with the typical hydrothermal vein-type uranium deposit, such as the Bois Noirs deposit in France [45]. The comparison between REE patterns measured in U oxides and those of granites shows that the fractionation between light and heavy REE in pitchblende from the Mianhuakeng deposit is more similar to that of the Changjiang pluton based on the $\sum$ LREE $/ \sum$ HREE and $(\mathrm{La} / \mathrm{Yb})_{\mathrm{N}}$ values that are 4.90 and 9.01 for pitchblende and 7.74 and 8.36 for the Changiiang pluton (Figure 7). However, the fractionation between light and heavy REE is relatively larger for Youdong pluton and smaller for the mafic dyke material (Figure 7). The flat pattern of LREE and HREE for pitchblende is more similar to the mafic dyke, although the Changjiang pluton also has a relatively flat signature of HREE (enrichment in HREE). A pronounced negative Eu anomaly is the biggest difference between pitchblende and the mafic dyke, but it is similar to both mineralization-bearing granites. 

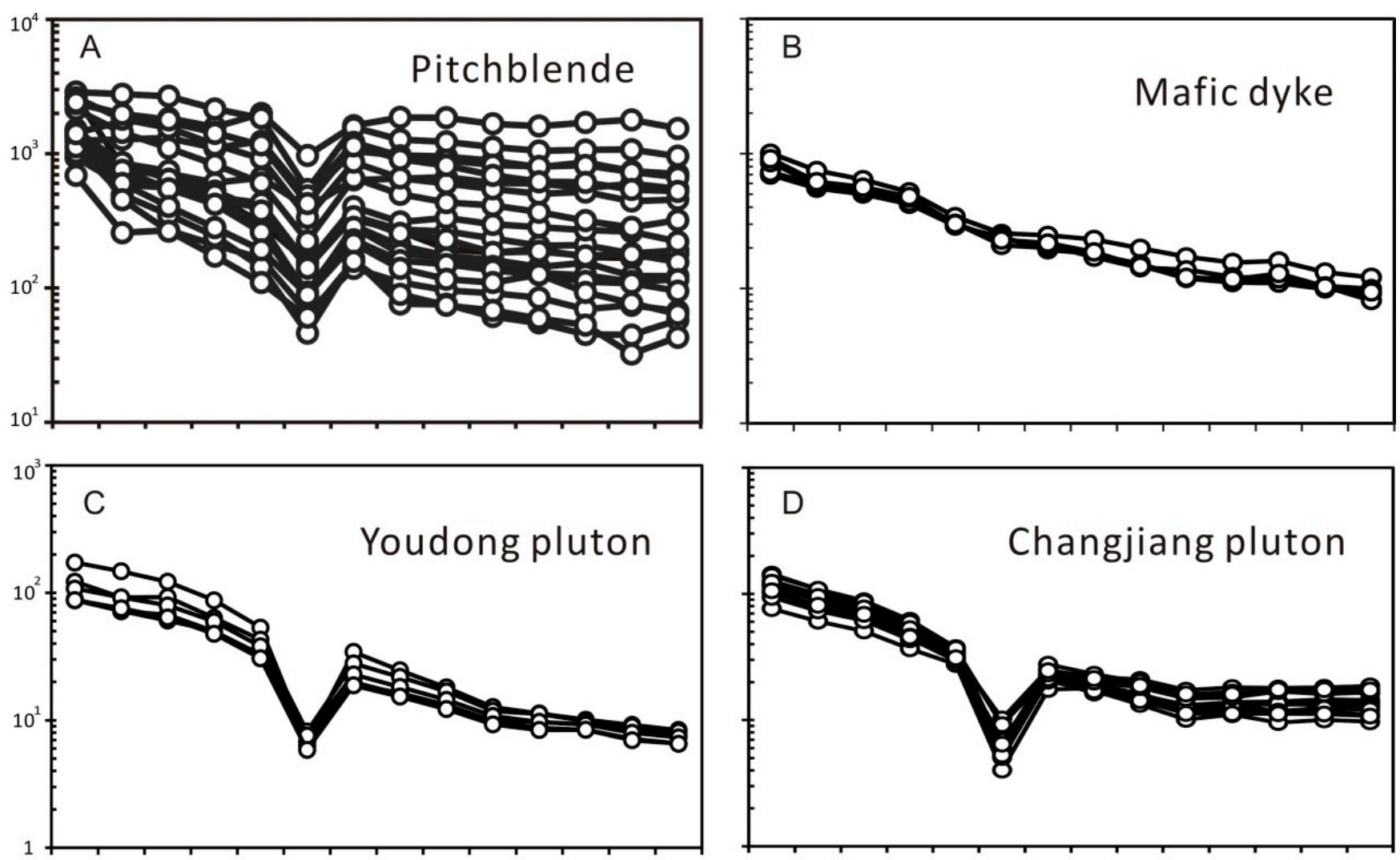

La Ce Pr Nd Sm Eu Gd Tb Dy Ho Er Tm Yb Lu

La Ce Pr Nd Sm Eu Gd Tb Dy Ho Er Tm Yb Lu

Figure 7. Chondrite-normalized REE patterns of (A) pitchblende from the Mianhuakeng deposit, (B) mafic dykes, (C) Indosinian Youdong granite, and (D) Yanshanian Changjiang granite. The REE data of mafic dykes, Youdong and Changjiang granite are from Zhan [32], Huang et al. [27] and Zhang et al. [29], respectively.

The $\delta^{34} S$ values of syn-mineralization pyrite range from -10.2 to $-1.4 \%$ o (average $-7.4 \%$ ), with the exception of one spot with a $\delta^{34} \mathrm{~S}$ value of $-13.2 \%$ (Table 3 ). Most of the $\delta^{34} \mathrm{~S}$ values for syn-mineralization pyrite are higher than those for pyrite from granites located near the studied area $\left(-10.9 \sim-7.1 \%\right.$ ) [48] and are lower than the $\delta^{34} S$ values $(-0.2 \sim-0.5 \%$ o ) of pyrite from diabase (Figure 8; [32]). Furthermore, the Changjiang pluton is a medium-grained biotite granite that contains an elevated $U$ content (31.9 81.7 ppm) and displays low Th/U ratios (1.0 1.6). These features are characteristic of a good source of $U$ for the mineralization [7]. Therefore, the major $U$ source for the Mianhuakeng $U$ deposit is most likely the Changjiang pluton based on REE patterns, sulfur isotopes, and trace element signatures, accompanied by the incorporation of mantle-derived fluids that accompanied the diabase intrusions. This conclusion is consistent with the mineralization ages that are in excellent agreement with the four episodes of mantle-derived mafic dykes, indicating the contribution of materials and heating sources from mafic magmatism and associated with coeval uranium mineralization. The close spatial relationship between $U$ mineralization and mafic dyke was also identified in other hydrothermal vein-type deposits worldwide [49]. Recently, scientific drilling in the study area has shown that thick and large enough $U$ deposits have been discovered at $-950 \mathrm{~m}$ below the surface, with the deepest minable $U$ deposit being found at $-1535 \mathrm{~m}$ below the surface [30]. Thus, there is a great metallogenic potential at depth in the study area. 


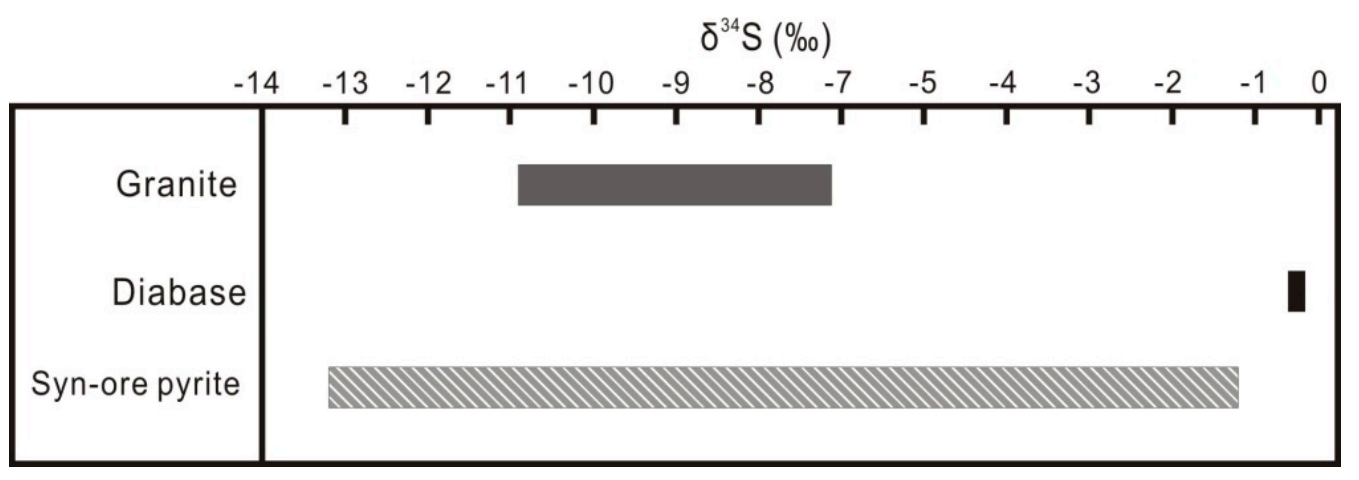

Figure 8. The $\delta^{34} \mathrm{~S}$ ranges of pyrites from granites and diabase, and from syn-mineralization pyrite in the Mianhuakeng $U$ deposit and its peripheral region. The sulfur isotope data of pyrites from granite and diabase were cited from $\mathrm{Hu}$ and Jin [48] and Zhan [32], respectively.

More than $90 \%$ of the outcrop area of the eastern Cathaysian block is covered by late Mesozoic volcanic and granitic rocks (Figure 1B; $[10,50,51])$. The U-rich mineralizationbearing granites likely have formed an important source for hydrothermal uranium mineralization in South China. Previous studies have shown that the Cretaceous tectonic regime of South China was dominated by extension [2,3,13,52,53], which resulted in the formation of numerous NE-SW trending grabens filled with red beds (Figure 1B) and the emplacement of mantle-derived mafic dikes (Figure 2; [13,52,54,55]). Field relations indicate that the uranium mineralization has good spatial correspondence with the emplacement of mafic dykes within the granite pluton. The parental magma of the mafic dykes is believed to have contributed significant amounts of $\mathrm{CO}_{2}$ to the mineralization-forming fluids $[13,52,56,57]$. The $\mathrm{CO}_{2}$ likely acted as a U-complexing agent and the circulation of $\mathrm{CO}_{2}$-rich hydrothermal fluids led to uranium being leached from granite source rocks, especially from the Changjiang pluton, and being transported as uranyl-carbonate complexes. The large fluctuations of $\mathrm{pH}$ values of the mineralization-forming fluid, probably resulting from $\mathrm{CO}_{2}$ effervescence or fluid phase separation, further led to decomposition of the uranyl carbonate complexes, and finally resulted in precipitation of the $U$ minerals in favorable structural traps to form the hydrothermal vein-type Mianhuakeng $U$ deposit.

\section{Conclusions}

The geochemical characteristics of syn-mineralization pyrite and pitchblende indicate that the mineralization-forming fluid in the Mianhuakeng $U$ deposit belongs to an intermediate to low temperature hydrothermal system. The pitchblende has REE signatures that are consistent with the typical hydrothermal vein-type $U$ deposit and the mineralization-forming fluid is determined to be reducing.

The $\delta^{34} S$ values of syn-mineralization pyrite are from -10.2 to $-1.4 \%$, which are located between those values of pyrite from granite near the studied area and the $\delta^{34} S$ values of pyrite from diabase in the ore district. The REE signatures (REE patterns and negative Eu anomaly) of pitchblende and sulfur isotope composition of syn-mineralization pyrite suggest that a major $U$ source for the Mianhuakeng $U$ deposit is most likely the Changjiang granite, accompanied by the incorporation of mantle-derived fluids.

The circulation of $\mathrm{CO}_{2}$-rich hydrothermal fluids leached uranium from the granite source rocks. The change of physicochemical properties of the mineralization-forming fluid finally resulted in the $U$ minerals precipitating in favorable structural traps to form the hydrothermal vein-type Mianhuakeng U deposit.

Author Contributions: Funding acquisition, J.L.; Writing—original draft, Q.L.; Writing—review \& editing, S.F. All authors have read and agreed to the published version of the manuscript.

Funding: This work was financially supported by jointly the projects of the National Key R\&D Program of China (2017YFC0602601), National Natural Science Foundation of China (41702101, 
42072098), West Light Foundation of the Chinese Academy of Science, and the Strategic Priority Research Program (B) of Chinese Academy of Sciences (Grant No. XDB18000000).

Data Availability Statement: Not applicable.

Acknowledgments: The authors are indebted to the colleagues from the Research Institute No. 290, Bureau of Geology of the Chinese National Nuclear Corporation in Shaoguan, Guangdong, for their field support and access to the mine sites.

Conflicts of Interest: The authors declare no conflict of interest.

\section{References}

1. Hu, R.Z.; Chen, W.T.; Xu, D.R.; Zhou, M.F. Reviews and new metallogenic models of mineral deposits in South China: An introduction. J. Asian Earth Sci. 2017, 137, 1-8. [CrossRef]

2. Hu, R.Z.; Zhou, M.F. Multiple Mesozoic mineralization events in South China-An introduction to the thematic issue. Miner. Depos. 2012, 47, 579-588. [CrossRef]

3. Mao, J.; Cheng, Y.; Chen, M.; Franco, P. Major types and time-space distribution of Mesozoic ore deposits in South China and their geodynamic settings. Miner. Depos. 2013, 48, 267-294.

4. Mao, J.; Pirajno, F.; Cook, N. Mesozoic metallogeny in East China and corresponding geodynamic settings-An introduction to the special issue. Ore Geol. Rev. 2011, 43, 1-7. [CrossRef]

5. Hua, R.M.; Chen, P.R.; Zhang, W.L.; Lu, J.J. Three major metallogenic events in Mesozoic in South China. Miner. Depos. 2005, 24, 99-107. (In Chinese)

6. Dahlkamp, F.J. Uranium Deposits of the World; Springer: Berlin/Heidelberg, Germany, 2009; pp. 1-493.

7. Bonnetti, C.; Liu, X.; Mercadier, J.; Cuney, M.; Deloule, E.; Villeneuve, J.; Liu, W. The genesis of granite-related hydrothermal uranium deposits in the Xiazhuang and Zhuguang ore fields, North Guangdong Province, SE China: Insights from mineralogical, trace elements and U-Pb isotopes signatures of the U mineralization. Ore Geol. Rev. 2018, 92, 588-612. [CrossRef]

8. Deng, P.; Ren, J.S.; Ling, H.F.; Shen, W.Z.; Sun, L.Q.; Zhu, B.; Tan, Z.Z. Yanshanian granite batholiths of southern Zhuguang Mountian: SHRIMP zircon U-Pb dating and tectonic implications. Geol. Rev. 2011, 57, 881-888. (In Chinese)

9. Deng, P.; Ren, J.S.; Ling, H.F.; Shen, W.Z.; Sun, L.Q. SHRIMP zircon U-Pb ages and tectonic implications for Indosinian granitoids of southern Zhuguangshan granitic composite, South China. Chin. Sci. Bull. 2012, 57, 1542-1552. [CrossRef]

10. Sun, T. A new map showing the distribution of granites in South China and its explanatory notes. Geol. Bull. China 2006, 25, 332-335.

11. Li, X.H.; Li, W.X.; Wang, X.C.; Li, Q.L.; Liu, Y.; Tang, G.Q.; Gao, Y.Y.; Wu, F.Y. SIMS U-Pb zircon geochronology of porphyry $\mathrm{Cu}-\mathrm{Au}-(\mathrm{Mo})$ deposits in the Yangtze River Metallogenic Belt, eastern China: Magmatic response to early Cretaceous lithospheric extension. Lithos 2010, 119, 427-438. [CrossRef]

12. Lan, Q.; Lin, J.; Fu, S.L.; Luo, J.C. Cathodoluminescent textures and trace element signatures of hydrothermal quartz from the granite-related No. 302 uranium deposit, South China: A reconnaissance study for their genetic significances. J. Geochem. Explor. 2021, 224, 106740. [CrossRef]

13. Hu, R.Z.; Bi, X.W.; Zhou, M.F.; Peng, J.T.; Su, W.C.; Liu, S.; Qi, H.W. Uranium Metallogenesis in South China and Its Relationship to Crustal Extension during the Cretaceous to Tertiary. Econ. Geol. 2008, 103, 583-598. [CrossRef]

14. Zhang, C.; Cai, Y.; Xu, H.; Dong, Q.; Liu, J.; Hao, R. Mechanism of mineralization in the Changjiang uranium ore field, South China: Evidence from fluid inclusions, hydrothermal alteration, and H-O isotopes. Ore Geol. Rev. 2017, 86, 225-253. [CrossRef]

15. Chen, P.R.; Liu, Y. The Physicochemical conditions for metallogenesis of No. 302 uranium deposit and the source and migration direction of its hydrothermal solution. Miner. Depos. 1990, 9, 149-157. (In Chinese)

16. Guo, G.L.; Liu, X.D.; Pan, J.Y.; Liu, C.D.; Yan, Z.B.; Chen, Y.P. Study of fluid inclusion from uranium deposit No. 302 in north Guangdong. Uranium Geol. 2010, 26, 350-368. (In Chinese)

17. Huang, G.L.; Yin, Z.P.; Ling, H.F.; Deng, P.; Zhu, B.; Shen, W.Z. Formation age, Geochemical characteristics and genesis of pitcheblende from No.302 uranium deposit in northern Guangdong. Miner. Depos. 2010, 29, 352-360. (In Chinese)

18. Jin, J.F.; Hu, R.Z. Transportation and deposition of uranium in the hydrothermal ore-forming fluids as exemplified by uranium deposit No. 302. Geochimica 1987, 4, 320-329. (In Chinese)

19. Qi, J.M.; Zhu, B.; Wu, J.Y.; Cao, H.J.; Liu, W.Q.; Xu, Z.Q. The evolution of ore-forming fluid and its constraint on mineralization process in Mianhuakeng uranium deposit, northern Guangdong, China. Acta Petrol. Sin. 2019, 35, 2711-2726.

20. Zhang, G.Q.; Hu, R.Z.; Shang, P.Q.; Tian, J.J.; Shuang, Y. Study on the C-O isotopic composition of calcites and metallogenic dynamics background in the No. 302 uranium deposit. Acta Mineral. Sin. 2008, 28, 413-420. (In Chinese)

21. Zhang, L.; Chen, Z.; Li, X.; Li, S.; Santosh, M.; Huang, G. Zircon U-Pb geochronology and geochemistry of granites in the Zhuguangshan complex, South China: Implications for uranium mineralization. Lithos 2018, 308, 19-33. [CrossRef]

22. Zhang, G.Q. Geochemistry of Hydrothermal Uranium Deposits in South China: A Case Study of the No. 302 Uranium Deposi. Ph.D. Thesis, University of Chinese Academy of Sciences, Beijing, China, 2008; pp. 1-94. (In Chinese).

23. Zhao, J.H.; Zhou, M.F.; Yan, D.P.; Zheng, J.P.; Li, J.W. Reappraisal of the ages of Neoproterozoic strata in South China: No connection with the Grenvillian orogeny. Geology 2011, 39, 299-302. [CrossRef] 
24. Charvet, J. The Neoproterozoic-Early Paleozoic tectonic evolution of the South China Block: An overview. J. Asian Earth Sci. 2013, 74, 198-209. [CrossRef]

25. Chen, J.F.; Jahn, B.M. Crustal evolution of southeastern China: Nd and Sr isotopic evidence. Tectonophysics 1998, $284,101-133$. [CrossRef]

26. Li, Z.X.; Li, X.H. Formation of the 1300-km-wide intracontinental orogen and postorogenic magmatic province in Mesozoic South China: A flat-slab subduction model. Geology 2007, 35, 179. [CrossRef]

27. Huang, G.L.; Cao, H.J.; Ling, H.F.; Shen, W.Z.; Wang, X.D.; Fu, S.C. Zircon SHRIMP U-Pb age, geochemistry and genesis of the Youdong granite in Northern Guangdong. Acta Geol. Sin. 2012, 86, 577-586. (In Chinese)

28. Huang, G.L.; Cao, H.J.; Xu, W.X.; Shen, W.Z. Vertical zoning model and prospecting potential in depth of Mianhuakeng uranium deposit in Zhuguang. Uranium Geol. 2015, 31, 355-362. (In Chinese)

29. Zhang, L.; Chen, Z.; Li, S.; Santosh, M.; Huang, G.; Tian, Z. Isotope geochronology, geochemistry, and mineral chemistry of the U-bearing and barren granites from the Zhuguangshan complex, South China: Implications for petrogenesis and uranium mineralization. Ore Geol. Rev. 2017, 91, 1040-1065. [CrossRef]

30. Li, Z.Y.; Qin, M.K.; Fan, H.H.; Cai, Y.Q.; Cheng, J.X.; Guo, D.F.; Ye, F.W.; Fan, G.; Liu, X.Y. Main Progresses of Uranium Geology and Exploration Techniques for the Past Decade in China. Bull. Mineral. Petrol. Geochem. 2020, 40, 1-13. (In Chinese)

31. Danyushevsky, L.; Robinson, P.; Gilbert, S.; Norman, M.; Large, R.; Mc Goldrick, P.; Shelley, M. Routine quantitative multi-element analysis of sulphide minerals by laser ablation ICP-MS: Standard development and consideration of matrix effects. Geochem. Explor. Environ. Anal. 2011, 11, 51-60. [CrossRef]

32. Zhan, L.G. The Geochemical Characteristics and Tectonic Stress Field of the No. 302 Uranium Deposit in Northern Guangdong Province. Ph.D. Thesis, East China University of Technology, Nanchang, China, 2016; pp. 1-95. (In Chinese).

33. Agangi, A.; Hofmann, A.; Wohlgemuth-Ueberwasser, C.C. Pyrite Zoning as a Record of Mineralization in the Ventersdorp Contact Reef, Witwatersrand Basin, South Africa. Econ. Geol. 2013, 108, 1243-1272. [CrossRef]

34. Daneshvar, N.; Maanijou, M.; Lentz, D.R.; Azizi, H.; McFarlane, C.R.M.; Maruoka, T. Interpretation of hydrothermal evolution in the Qolqoleh gold deposit, southwest of Saqqez, Iran: Analysis of pyrite by LA-ICP-MS and sulfur isotopes. Ore Geol. Rev. 2021, 133, 104087. [CrossRef]

35. Dehnavi, A.S.; McFarlane, C.R.M.; Lentz, D.R.; Walker, J.A. Assessment of pyrite composition by LA-ICP-MS techniques from massive sulfide deposits of the Bathurst Mining Camp, Canada: From textural and chemical evolution to its application as a vectoring tool for the exploration of VMS deposits. Ore Geol. Rev. 2018, 92, 656-671. [CrossRef]

36. Large, R.R.; Danyushevsky, L.; Hollit, C.; Maslennikov, V.; Meffre, S.; Gilbert, S.; Bull, S.; Scott, R.; Emsbo, P.; Thomas, H.; et al. Gold and Trace Element Zonation in Pyrite Using a Laser Imaging Technique: Implications for the Timing of Gold in Orogenic and Carlin-Style Sediment-Hosted Deposits. Econ. Geol. 2009, 104, 635-668. [CrossRef]

37. Large, R.R.; Mukherjee, I.; Gregory, D.; Steadman, J.; Corkrey, R.; Danyushevsky, L.V. Atmosphere oxygen cycling through the Proterozoic and Phanerozoic. Miner. Depos. 2019, 54, 485-506. [CrossRef]

38. Maslennikov, V.V.; Maslennikova, S.P.; Large, R.R.; Danyushevsky, L.V. Study of Trace Element Zonation in Vent Chimneys from the Silurian Yaman-Kasy Volcanic-Hosted Massive Sulfide Deposit (Southern Urals, Russia) Using Laser Ablation-Inductively Coupled Plasma Mass Spectrometry (LA-ICPMS). Econ. Geol. 2009, 104, 1111-1141. [CrossRef]

39. Cook, N.J.; Ciobanu, C.L.; Meria, D.; Silcock, D.; Wade, B. Arsenopyrite-Pyrite Association in an Orogenic Gold Ore: Tracing Mineralization History from Textures and Trace Elements. Econ. Geol. 2013, 108, 1273-1283. [CrossRef]

40. Ballantyne, J.M.; Moore, J.N. Arsenic geochemistry in geothermal systems. Geochim. Cosmochim. Acta 1988, 52, 475-483. [CrossRef]

41. Reich, M.; Deditius, A.; Chryssoulis, S.; Li, J.W.; Ma, C.Q.; Parada, M.A.; Barra, F.; Mittermayr, F. Pyrite as a record of hydrothermal fluid evolution in a porphyry copper system: A SIMS/EMPA trace element study. Geochim. Cosmochim. Acta 2013, $104,42-62$. [CrossRef]

42. Sheng, X.; Bi, X.; Hu, R.; Tang, Y.; Lan, Q.; Xiao, J.; Tao, Y.; Huang, M.; Peng, J.; Xu, L. The mineralization process of the Lanuoma $\mathrm{Pb}-\mathrm{Zn}-\mathrm{Sb}$ deposit in the Sanjiang Tethys region: Constraints from in situ sulfur isotopes and trace element compositions. Ore Geol. Rev. 2019, 111, 102941. [CrossRef]

43. Goodfellow, W.D.; Jonasson, I.R.; Morganti, J.M. Zonation of chalcophile elements about the howard's pass (XY) Zn-Pb deposit, Selwyn Basin, Yukon. J. Geochem. Explor. 1983, 19, 503-542. [CrossRef]

44. Thomas, H.V.; Large, R.R.; Bull, S.W.; Maslennikov, V.; Berry, R.F.; Fraser, R.; Froud, S.; Moye, R. Pyrite and Pyrrhotite Textures and Composition in Sediments, Laminated Quartz Veins, and Reefs at Bendigo Gold Mine, Australia: Insights for Ore Genesis. Econ. Geol. 2011, 106, 1-31. [CrossRef]

45. Mercadier, J.; Cuney, M.; Lach, P.; Boiron, M.C.; Bonhoure, J.; Richard, A.; Leisen, M.; Kister, P. Origin of uranium deposits revealed by their rare earth element signature. Terra Nova 2011, 23, 264-269. [CrossRef]

46. Hu, R.Z.; Jin, J.F. Mechanism of the migration and deposition of uranium in ascending hydrothermal solutions: Evidence from the Xiwang uranium deposit. Geol. Rev. 1990, 36, 317-325. (In Chinese)

47. Fryer, B.J.; Taylor, R.P. Rare-earth element distributions in uraninites: Implications for ore genesis. Chem. Geol. 1987, 63, 101-108. [CrossRef]

48. Hu, R.Z.; Jin, J.F. Genesis and origin of the Guidong granitic batholith. J. Chendu Coll. Geol. 1988, 15, 20-28. (In Chinese)

49. Leroy, J. The Margnac and Fanay uranium deposits of the La Crouzille District (western Massif Central, France); geologic and fluid inclusion studies. Econ. Geol. 1978, 73, 1611-1634. [CrossRef] 
50. Gilder, S.A.; Gill, J.; Coe, R.S.; Zhao, X.; Liu, Z.; Wang, G.; Yuan, K.; Liu, W.; Kuang, G.; Wu, H. Isotopic and paleomagnetic constraints on the Mesozoic tectonic evolution of south China. J. Geophys. Res. 1996, 101, 16137-16154. [CrossRef]

51. Zhou, X.M. Genesis of the Late Mesozoic Granites at Nanling: Implications to Lithosphere Dynamic Evolution; Science Publishing House: Beijing, China, 2007.

52. Hu, R.Z.; Burnard, P.G.; Bi, X.W.; Zhou, M.F.; Peng, J.T.; Su, W.C.; Zhao, J.-H. Mantle-derived gaseous components in ore-forming fluids of the Xiangshan uranium deposit, Jiangxi province, China: Evidence from He, Ar and C isotopes. Chem. Geol. 2009, 266, 86-95. [CrossRef]

53. Liu, H.; Tang, L.; Kao, J. Evolution and Structural Analysis of the Mesozoic and Cenozoic Rift System in Asia: Scientific Papers on Geology for Scientific Exchange. In Proceedings of the International Geological Congress, Moscow, Russia, 4-14 August 1984; pp. 57-67.

54. Li, X.H.; Hu, R.Z.; Rao, B. Geochronology and geochemistry of Cretaceous mafic dikes from northern Guangdong, SE China. Geochimica 1997, 26, 14-31. (In Chinese)

55. Qi, Y.; Hu, R.; Liu, S.; Coulson, I.M.; Qi, H.; Tian, J.; Feng, C.; Wang, T. Geochemical and Sr-Nd-Pb isotopic compositions of Mesozoic mafic dikes from the Gan-Hang tectonic belt, South China: Petrogenesis and geodynamic significance. Int. Geol. Rev. 2012, 54, 920-939. [CrossRef]

56. Cuney, M. The extreme diversity of uranium deposits. Miner. Depos. 2009, 44, 3-9. [CrossRef]

57. Min, M.Z.; Luo, X.Z.; Du, G.S.; He, B.A.; Campbell, A.R. Mineralogical and geochemical constraints on the genesis of the granite-hosted Huangao uranium deposit, SE China. Ore Geol. Rev. 1999, 14, 105-127. [CrossRef] 